\title{
On a Class of Nonlinear Convolution Equations
}

\author{
L. v. Wolfersdorf and J. Janno \\ With reverence dedicated to Prof. Dr. H. Beckert \\ on the occasion of his 75 th birthday
}

\begin{abstract}
By means of weighted norms existence and uniqueness theorems are proved for some classes of nonlinear convolution equations in Lebesgue spaces $L_{p}$ and spaces $C$ of continuous functions. The applicability of the theorems is shown by examples.

Keywords: Nonlinear convolution equations, Volterra integral equations, integro-differential equations
\end{abstract}

AMS subject classification: $45 \mathrm{D} 05,45 \mathrm{G} 10,45 \mathrm{~J} 05,45 \mathrm{~N} 05$

\section{Introduction}

In the theory of inverse problems for identifying memory kernels in viscoelasticity and heat transfer a class of nonlinear convolution equations occurs. Recently for these equations global existence theorems are proved using weighted norms (see $[1,6,8])$. In particular Bukhgeim [1] derives general theorems for corresponding nonlinear Volterra equations with a convolution majorant by means of Schauder fixed point theorem and the contraction principle in Lebesgue spaces $L_{p}$ and spaces $C$ of continuous functions.

In the present paper such existence theorem is given for some general classes of nonlinear convolution equations in spaces $L_{p}$ and $C$ applying the contraction principle in a modified way. This extends the existence lemma used in [8]. Further some examples are mentioned showing the applicability of the theorem, among them equations of auto-convolution type, nonlinear stress-strain relations in viscoelasticity and nonlinear Volterra integro-differential equations.

\section{Preparations}

Let $X$ be a normalized, commutative, separable Banach algebra with norm $\|\cdot\|$ and $L_{p}(0, T ; X) \quad(1 \leq p \leq \infty)$ the spaces of $X$-valued functions with the norms

$$
\|u\|_{p}=\left(\int_{0}^{T}\|u(t)\|^{p} d t\right)^{1 / p} \quad(1 \leq p<\infty) \quad \text { and } \quad\|u\|_{\infty}=\sup _{t \in[0, T]} \operatorname{ess}\|u(t)\|
$$

L. v. Wolfersdorf: Freiberg Univ. Mining Techn., Fac. Math. Comp. Sci., D - 09596 Freiberg J. Janno: Estonian Acad. Sci., Inst. Cyb., EE - 0026 Tallinn, Estonia 
respectively. For $0<T<\infty$ in these spaces we introduce the equivalent weighted norms

$$
\|u\|_{p, \sigma}=\left\|e^{-\sigma t} u\right\|_{p} \quad(\sigma \geq 0)
$$

which satisfy the relations

$$
\|u\|_{p, \sigma} \leq\|u\|_{p} \leq e^{\sigma T}\|u\|_{p, \sigma} .
$$

The convolution operator

$$
(u * v)(t)=\int_{0}^{t} u(t-s) v(s) d s
$$

in these spaces is commutative and invariant with respect to multiplication by $e^{-\sigma t}$. From the Young inequality in the weighted norms $\|u * v\|_{p, \sigma} \leq\|u\|_{1, \sigma}\|v\|_{p, \sigma}$ and the estimates

$$
\|u\|_{1, \sigma} \leq\|1\|_{q}\|u\|_{p, \sigma} \quad \text { and } \quad\|u\|_{1, \sigma} \leq\left\|e^{-\sigma t}\right\|_{q}\left\|_{u}\right\|_{p} \quad\left(\frac{1}{p}+\frac{1}{q}=1\right)
$$

following from Hölder's inequality, we have the estimations

$$
\|u * v\|_{p, \sigma} \leq T^{1 / q}\|u\|_{p, \sigma}\|v\|_{p, \sigma}
$$

and

$$
\|u * v\|_{p, \sigma} \leq\left(\frac{1}{q \sigma}\right)^{1 / q}\|u\|_{p}\|v\|_{p, \sigma} \leq\left(\frac{1}{q \sigma}\right)^{1 / q}\|u\|_{p}\|v\|_{p}
$$

where $p>1$. Further we define the set of functions

$$
\mathcal{M}=\left\{M \in\left(\mathbb{R}_{+}^{2} \rightarrow \mathbb{R}_{+}\right) \mid \begin{array}{l}
M \text { is non-decreasing with respect } \\
\text { to each component of its argument }
\end{array}\right\}
$$

\section{Main result}

Our main result is an existence and uniqueness statement for second kind operator equations of the form

$$
u+G_{0} u+G_{1} u * G_{2} u=g
$$

where $G_{i}(i=0,1,2)$ are operators in the spaces $L_{p}(0, T ; X)$ and $C(0, T ; X)$ satisfying Lipschitz conditions in the weighted norms. More precisely, there holds the following 
Theorem. Let $G_{i} \in\left(L_{p}(0, T ; X) \rightarrow L_{p}(0, T ; X)\right) \quad(i=0,1,2)$, where $0<T<\infty$ and $p>1$, satisfy Lipschitz conditions of the form

$$
\left\|G_{i} u-G_{i} v\right\|_{p, \sigma} \leq M_{i}\left(\|u\|_{p, \sigma},\|v\|_{p, \sigma}\right)\|u-v\|_{p, \sigma} \quad\left(\sigma \geq \sigma_{0}>0\right)
$$

for $i=1,2$ and

$$
\left\|G_{0} u-G_{0} v\right\|_{p, \sigma} \leq \lambda(\sigma) M_{0}\left(\|u\|_{p, \sigma},\|v\|_{p, \sigma}\right)\|u-v\|_{p, \sigma} \quad\left(\sigma \geq \sigma_{0}>0\right)
$$

where $M_{i} \in \mathcal{M}(i=0,1,2)$ and $\lambda$ is a non-increasing continuous function with $\lambda(\sigma) \rightarrow$ 0 as $\sigma \rightarrow \infty$.

Then equation (4) has a unique solution $u \in L_{p}(0, T ; X)$ for any $g \in L_{p}(0, T ; X)$. The same statement holds for the space $C(0, T ; X)$.

Proof. 1. At first we consider the auxiliary equation

$$
f+G_{0} f=g,
$$

i.e. the special case of equation (4) without convolution term. By contraction principle we show the existence of a solution to equation (7) in the ball $B_{\rho, \sigma}(g)=\left\{f:\|f-g\|_{p, \sigma} \leq\right.$ $\rho\}$, where $\rho=2\left\|G_{0} g\right\|_{p}$ and $\sigma \geq \sigma_{0}$ is chosen as a solution of the equation

$$
\lambda(\sigma) M_{0}\left(\rho+\|g\|_{p}, \rho+\|g\|_{p}\right)=\varepsilon
$$

with some $\varepsilon \in\left(0, \frac{1}{2}\right.$ ). Due to the assumptions on $\lambda$ a solution $\sigma$ of equation (8) exists for any sufficiently small positive $\varepsilon$. Further by (6) and (8) for the operator $A_{0} f=g-G_{0} f$ in $B_{p, \sigma}(g)$ we have the estimates

$$
\begin{aligned}
\left\|A_{0} f_{1}-A_{0} f_{2}\right\|_{p, \sigma} & =\left\|G_{0} f_{1}-G_{0} f_{2}\right\|_{p, \sigma} \\
& \leq \lambda(\sigma) M_{0}\left(\left\|f_{1}\right\|_{p, \sigma},\left\|f_{2}\right\|_{p, \sigma}\right)\left\|f_{1}-f_{2}\right\|_{p, \sigma} \\
& \leq \lambda(\sigma) M_{0}\left(\rho+\|g\|_{p, \sigma}, \rho+\|g\|_{p, \sigma}\right)\left\|f_{1}-f_{2}\right\|_{p, \sigma} \\
& \leq \varepsilon\left\|f_{1}-f_{2}\right\|_{p, \sigma}
\end{aligned}
$$

so that $A_{0}$ is a contraction. Moreover,

$$
\begin{aligned}
\left\|A_{0} f-g\right\|_{p, \sigma} & =\left\|G_{0} f\right\|_{p, \sigma} \\
& \leq\left\|G_{0} f-G_{0} g\right\|_{p, \sigma}+\left\|G_{0} g\right\|_{p, \sigma} \\
& \leq \lambda(\sigma) M_{0}\left(\rho+\|g\|_{p, \sigma},\|g\|_{p, \sigma}\right)\|f-g\|_{p, \sigma}+\left\|G_{0} g\right\|_{p, \sigma} \\
& \leq\left(\varepsilon+\frac{1}{2}\right) \rho \\
& \leq \rho
\end{aligned}
$$

so that $A_{0}$ maps $B_{\rho, \sigma}(g)$ into itself.

2. Next we are going to show that a unique solution of equation (4) exists in the ball $B_{\rho, \sigma}(f)=\left\{u:\|u-f\|_{p, \sigma} \leq \rho\right\}$ with some $\rho$ and $\sigma$, also using the contraction principle. In view of equation (7) the operator $A u=g-G_{0} u-G_{1} u * G_{2} u$ writes

$$
\begin{aligned}
f-A u= & G_{1} u * G_{2} u+G_{0} u-G_{0} f \\
= & \left(G_{1} u-G_{1} f\right) *\left(G_{2} u-G_{2} f\right)+G_{1} f *\left(G_{2} u-G_{2} f\right) \\
& +\left(G_{1} u-G_{1} f\right) * G_{2} f+G_{1} f * G_{2} f+G_{0} u-G_{0} f .
\end{aligned}
$$


Making use of the inequalities (2) and (3), we obtain

$$
\begin{aligned}
\|f-A u\|_{p, \sigma} \leq & T^{1 / q}\left\|G_{1} u-G_{1} f\right\|_{p, \sigma}\left\|G_{2} u-G_{2} f\right\|_{p, \sigma} \\
& +\left(\frac{1}{q \sigma}\right)^{1 / q}\left\|G_{1} f\right\|_{p}\left\|G_{2} u-G_{2} f\right\|_{p, \sigma} \\
& +\left(\frac{1}{q \sigma}\right)^{1 / q}\left\|G_{2} f\right\|_{p}\left\|G_{1} u-G_{1} f\right\|_{p, \sigma} \\
& +\left(\frac{1}{q \sigma}\right)^{1 / q}\left\|G_{1} f\right\|_{p}\left\|G_{2} f\right\|_{p}+\left\|G_{0} u-G_{0} f\right\|_{p, \sigma} .
\end{aligned}
$$

Further, by the assumptions (5) and (6) we have

$$
\begin{aligned}
\|f-A u\|_{p, \sigma} \leq & T^{1 / q} M_{1}\left(\|f\|_{p, \sigma}+\|u-f\|_{p, \sigma},\|f\|_{p, \sigma}\right) \\
& \times M_{2}\left(\|f\|_{p, \sigma}+\|u-f\|_{p, \sigma},\|f\|_{p, \sigma}\right)\|u-f\|_{p, \sigma}^{2} \\
& +\left(\frac{1}{q \sigma}\right)^{1 / q}\left(\left\|G_{1} f\right\|_{p} M_{2}\left(\|f\|_{p, \sigma}+\|u-f\|_{p, \sigma},\|f\|_{p, \sigma}\right)\right. \\
& \left.+\left\|G_{2} f\right\|_{p} M_{1}\left(\|f\|_{p, \sigma}+\|u-f\|_{p, \sigma},\|f\|_{p, \sigma}\right)\right)\|u-f\|_{p, \sigma} \\
& +\left(\frac{1}{q \sigma}\right)^{1 / q}\left\|G_{1} f\right\|_{p}\left\|G_{2} f\right\|_{p} \\
& +\lambda(\sigma) M_{0}\left(\|f\|_{p, \sigma}+\|u-f\|_{p, \sigma},\|f\|_{p, \sigma}\right)\|u-f\|_{p, \sigma} .
\end{aligned}
$$

Now we choose $\rho_{1}>0$ and $\sigma_{1}(\rho) \geq \sigma_{0}$ such that there hold the estimations

$$
T^{1 / q} M_{1}\left(\|f\|_{p}+\rho,\|f\|_{p}\right) M_{2}\left(\|f\|_{p}+\rho,\|f\|_{p}\right) \rho \leq \alpha \quad(\alpha \in(0,1))
$$

and

$$
\begin{aligned}
& \left(\frac{1}{q \sigma}\right)^{1 / q}\left(\left\|G_{1} f\right\|_{p} M_{2}\left(\|f\|_{p}+\rho,\|f\|_{p}\right)+\left\|G_{2} f\right\|_{p} M_{1}\left(\|f\|_{p}+\rho,\|f\|_{p}\right)\right) \rho \\
+ & \left(\frac{1}{q \sigma}\right)^{1 / q}\left\|G_{1} f\right\|_{p}\left\|G_{2} f\right\|_{p}+\lambda(\sigma) M_{0}\left(\|f\|_{p}+\rho,\|f\|_{p}\right) \rho \leq(1-\alpha) \rho
\end{aligned}
$$

provided $\rho \leq \rho_{1}$ and $\sigma \geq \sigma_{1}(\rho)$. On account of the inclusions $M_{i} \in \mathcal{M}(i=0,1,2)$ it then follows that

$$
\|f-A u\|_{p, \sigma} \leq \rho
$$

if $u \in B_{\rho, \sigma}(f), \rho \leq \rho_{1}$ and $\sigma \geq \sigma_{1}(\rho)$. I.e., $A$ maps the ball $B_{\rho, \sigma}(f)$ into itself.

For the difference of the operator $A$ we write

$$
\begin{aligned}
A u_{2}-A u_{1}= & G_{1} u_{1} * G_{2} u_{1}+G_{0} u_{1}-G_{1} u_{2}+G_{2} u_{2}-G_{0} u_{2} \\
= & \left(G_{1} u_{1}-G_{1} u_{2}\right) *\left(G_{2} u_{1}-G_{2} f\right)+\left(G_{1} u_{1}-G_{1} u_{2}\right) * G_{2} f \\
& +\left(G_{1} u_{2}-G_{1} f\right) *\left(G_{2} u_{1}-G_{2} u_{2}\right) \\
& +G_{1} f *\left(G_{2} u_{1}-G_{2} u_{2}\right)+G_{0} u_{1}-G_{0} u_{2} .
\end{aligned}
$$


Estimating as above, for $u_{1}, u_{2} \in B_{\rho, \sigma}(f)$ we obtain

$$
\begin{aligned}
\| A u_{1}- & A u_{2} \|_{p, \sigma} \\
\leq & \left\{M_{1}\left(\|f\|_{p, \sigma}+\rho,\|f\|_{p, \sigma}+\rho\right)\right. \\
& \times\left(M_{2}\left(\|f\|_{p, \sigma}+\rho,\|f\|_{p, \sigma}\right) T^{1 / q} \rho+\left(\frac{1}{q \sigma}\right)^{1 / q}\left\|G_{2} f\right\|_{p}\right) \\
& +M_{2}\left(\|f\|_{p, \sigma}+\rho,\|f\|_{p, \sigma}+\rho\right) \\
& \times\left(M_{1}\left(\|f\|_{p, \sigma}+\rho,\|f\|_{p, \sigma}\right) T^{1 / q} \rho+\left(\frac{1}{q \sigma}\right)^{1 / q}\left\|G_{1} f\right\|_{p}\right) \\
& \left.+\lambda(\sigma) M_{0}\left(\|f\|_{p, \sigma}+\rho,\|f\|_{p, \sigma}+\rho\right)\right\}\left\|u_{1}-u_{2}\right\|_{p, \sigma} \quad\left(\sigma \geq \sigma_{0}\right) .
\end{aligned}
$$

This time we choose $\rho_{2}>0$ and $\sigma_{2} \geq \sigma_{0}$ such that

$$
\begin{aligned}
M_{1}\left(\|f\|_{p}+\rho,\|f\|_{p}+\rho\right) & \left(M_{2}\left(\|f\|_{p}+\rho,\|f\|_{p}\right) T^{1 / q} \rho+\left(\frac{1}{q \sigma}\right)^{1 / q}\left\|G_{2} f\right\|_{p}\right) \\
+M_{2}\left(\|f\|_{p}+\rho,\|f\|_{p}+\rho\right)( & \left.M_{1}\left(\|f\|_{p}+\rho,\|f\|_{p}\right) T^{1 / q} \rho+\left(\frac{1}{q \sigma}\right)^{1 / q}\left\|G_{1} f\right\|_{p}\right) \\
& +\lambda(\sigma) M_{0}\left(\|f\|_{p}+\rho,\|f\|_{p}+\rho\right) \leq \mu<1
\end{aligned}
$$

provided $\rho \leq \rho_{2}$ and $\sigma \geq \sigma_{2}$. Then it follows that

$$
\left\|A u_{1}-A u_{2}\right\|_{p, \sigma} \leq \mu\left\|u_{1}-u_{2}\right\|_{p, \sigma} \quad\left(u_{1}, u_{2} \in B_{\rho, \sigma}(f)\right)
$$

if $\rho \leq \rho_{2}$ and $\sigma \geq \sigma_{2}$.

The estimations (9) and (10) show that the operator $A$ is a contraction in $B_{\rho, \sigma}(f)$ with $\rho \leq \rho_{3}=\min \left\{\rho_{1}, \rho_{2}\right\}$ and $\sigma \geq \sigma_{3}(\rho)=\max \left\{\sigma_{1}(\rho), \sigma_{2}\right\}$. Hence equation (4) has a unique solution in every ball $B_{\rho, \sigma}(f) \quad\left(\rho \leq \rho_{3}\right.$ and $\left.\sigma \geq \sigma_{3}(\rho)\right)$.

3. It remains to prove uniqueness in the whole space $L_{p}(0, T ; X)$. For this aim let $u$ be an arbitrary solution of equation (4) in $L_{p}(0, T ; X)$. From equations (4) and (7) we obtain

$$
u-f=G_{0} f-G_{0} u-G_{1} u * G_{2} u .
$$

Estimating by means of (6) and (3), we deduce the inequality

$$
\|u-f\|_{p, \sigma} \leq \dot{\lambda}(\sigma) M_{0}\left(\|f\|_{p},\|u\|_{p}\right)\|u-f\|_{p, \sigma}+\left(\frac{1}{q \sigma}\right)^{1 / q}\left\|G_{1} u\right\|_{p}\left\|G_{2} u\right\|_{p}
$$

which due to the convergence $\lambda(\sigma) \rightarrow 0$ as $\sigma \rightarrow \infty$ implies that $\|u-f\|_{p, \sigma} \rightarrow 0$ as $\sigma \rightarrow \infty$. This means, every solution $u \in L_{p}(0, T ; X)$ of equation (4) belongs to some ball $B_{\rho, \sigma}(f)$ with $\rho \leq \rho_{3}$ and sufficiently large $\sigma \geq \sigma_{3}(\rho)$, in which uniqueness of the solution has already been shown

Remark. The solution of equation (4) depends (locally Lipschitz-) continuously in the norm $\|\cdot\|_{p}$ on the data $g$ :

$$
\left\|u_{1}-u_{2}\right\|_{p} \leq M\left(T,\left\|G_{1} u_{1}\right\|_{p},\left\|G_{2} u_{2}\right\|_{p},\left\|u_{1}\right\|_{p},\left\|u_{2}\right\|_{p}\right)\left\|g_{1}-g_{2}\right\|_{p}
$$

where $M$ is a (continuous) function non-decreasing in its arguments. 


\section{Additional statements}

There are four corollaries to the Theorem. Since the convolution $G_{1} * G_{2}$ satisfies a Lipschitz condition of form (5) if $G_{1}$ and $G_{2}$ do it, we have at first the following

Corollary 1. The statements of the Theorem generally hold for equations

$$
u+G_{0} u+\sum_{i=1}^{n}\left(G_{i, 1} u * G_{i, 2} u * \ldots * G_{i, i+1} u\right)=g
$$

where

$$
G_{i, j} \in\left(L_{p}(0, T ; X) \rightarrow L_{p}(0, T ; X)\right)
$$

$(i=1, \ldots, n ; j=1, \ldots, i+1 ; n \in \mathbb{N})$ fulfill the Lipschitz condition (5) and

$$
G_{0} \in\left(L_{p}(0, T ; X) \rightarrow L_{p}(0, T ; X)\right)
$$

fulfills the Lipschitz condition (6).

Immediately from the proof of the Theorem there follows also

Corollary 2. For $k_{i} \in L_{\infty}([0, T] \times[0, T] ; X)$ or $k_{i} \in C([0, T] \times[0, T] ; X) \quad(i=$ $1, \ldots, n)$ with $\left\|k_{i}\right\|(t, s) \leq K_{i}=$ const the statements of the Theorem hold true for equations

$$
u+G_{0} u+\sum_{i=1}^{n} \int_{0}^{t} k_{i}(t, s) G_{i, 1} u(t-s) G_{i, 2} u(s) d s=g
$$

where $G_{0}$ and $G_{i, j} \quad(i=1, \ldots n ; j=1,2)$ are as in Corollary 1.

In comparison with Theorem 2.2 of Bukhgeim [1] we state the following condition

Corollary 3. Let $G_{0} \in\left(L_{p}(0, T ; X) \rightarrow L_{p}(0, T ; X)\right) \quad(p>1)$ fulfill the Lipschitz

$$
\left\|G_{0} u-G_{0} v\right\|_{p, \sigma} \leq \Psi\left(\|u\|_{p, \sigma}\|v\|_{p, \sigma}, \sigma\right)\|u-v\|_{p, \sigma} \quad\left(\sigma \geq \sigma_{0}>0\right)
$$

where the function $\Psi(r, \rho, \sigma)$ is non-decreasing in $r, \rho$ and non-increasing in $\sigma$ with

$$
\lim _{\sigma \rightarrow \infty} \Psi(r, r, \sigma)<1
$$

for some $r=r_{0}>0$. Then equation

$$
u+G_{0} u=g
$$

(see (7)) has a solution $u \in L_{p}^{\prime}(0, T ; X)$ for any function $g \in L_{p}(0, T ; X)$ which satisfies the condition

$$
\|h\|_{p, \sigma} \equiv\left\|g-g_{0}-G_{0} g_{0}\right\|_{p, \sigma} \rightarrow 0 \quad \text { as } \quad \sigma \rightarrow \infty
$$

with some function $g_{0} \in L_{p}(0, T ; X)$, provided $r_{0}>\left\|g_{0}\right\|_{p}$. In case $p<\infty$ condition (15) is fulfilled for any $g, g_{0} \in L_{p}(0, T ; X)$, in particular for $g_{0}=0$. In the case $p=\infty$ 
condition (15) is fulfilled for $g_{0}=g$ if $\lim _{t \rightarrow+0}\left\|G_{0} g\right\|(t)=0$. If condition (14) is fulfilled for any $r \geq\left\|g_{0}\right\|_{p}$, then the solution $u$ is unique in $L_{p}(0, T ; X)$.

Proof. The existence of a solution follows from the estimations

$$
\begin{aligned}
\left\|A_{0} u_{1}-A_{0} u_{2}\right\|_{p, \sigma} & \leq \Psi\left(\left\|g_{0}\right\|_{p}+\rho,\left\|g_{0}\right\|_{p}+\rho, \sigma\right)\left\|u_{1}-u_{2}\right\|_{p, \sigma} \\
\left\|A_{0} u-g_{0}\right\|_{p, \sigma} & \leq \Psi\left(\left\|g_{0}\right\|_{p}+\rho,\left\|g_{0}\right\|_{p}, \sigma\right) \rho+\|h\|_{p, \sigma}
\end{aligned}
$$

for the operator $A_{0} u=g-G_{0} u$ in the ball $B_{\rho, \sigma}\left(g_{0}\right)$, where $\rho$ and $\sigma$ are chosen such that

$$
\Psi\left(\left\|g_{0}\right\|_{p}+\rho,\left\|g_{0}\right\|_{p}+\rho, \sigma\right)<1 \quad \text { and } \quad\|h\|_{p, \sigma} \leq\left(1-\Psi\left(\left\|g_{0}\right\|+\rho,\left\|g_{0}\right\|, \sigma\right)\right) \rho .
$$

The uniqueness of the solution $u$ can be obtained from the estimation

$$
\left\|u-g_{0}\right\|_{p, \sigma} \leq \Psi\left(\|u\|_{p, \sigma},\left\|g_{0}\right\|_{p, \sigma}, \sigma\right)\left\|u-g_{0}\right\|_{p, \sigma}+\|h\|_{p, \sigma}
$$

as in the proof of the Theorem using (14)

The proof of the Theorem also shows the validity of the following

Corollary 4. The statements of the Theorem hold true for equation

$$
u+G_{0} u+F\left(G_{1} u * G_{2} u\right)=g
$$

where $G_{i}(i=1,2,3)$ are as in the Theorem and $F \in\left(L_{p}(0, T ; X) \rightarrow L_{p}(0, T ; X)\right)$ satisfies the assumptions $F 0=0$ and

$$
\left\|F v_{1}-F v_{2}\right\|_{p, \sigma} \leq M\left(\left\|v_{1}\right\|_{p, \sigma},\left\|v_{2}\right\|_{p, \sigma}\right)\left\|v_{1}-v_{2}\right\|_{p, \sigma}
$$

with $M \in \mathcal{M}$.

\section{Examples}

We illustrate the applicability of the Theorem and Corollaries $1-4$ by a few examples.

Example 1. At first we consider the nonlinear integral equation of generalized convolution type

$$
u(t)+\int_{0}^{t} k_{1}(t, s) u(s) d s+\int_{0}^{1} k_{2}(t, s) F_{1}(u(t-s)) F_{2}(u(s)) d s=g(t)
$$

in the spaces $L_{p}(0, T) \quad(p>1)$ and $C[0, T]$. In the literature, as a rule (see [5]), the special case of equation (18) without factor $F_{1}$ is treated. From Corollary 2 with $n=2$ we obtain existence and uniqueness of a solution $u$ in $L_{p}(0, T)$ or $C[0, T]$ for any function $g$ in $L_{p}(0, T)$ or $C[0, T]$, respectively, if

$$
k_{i} \in L_{\infty}([0, T] \times[0, T]) \quad \text { or } \quad k_{i} \in C([0, T] \times[0, T]) \quad(i=1,2)
$$


and $F_{i}$ satisfy the Lipschitz conditions

$$
\left|F_{i}(u)-F_{2}(v)\right| \leq N_{i}|u-v| \quad(u, v \in \mathbb{R})
$$

with constants $N_{i}(i=1,2)$.

In the special cases $k_{1} \equiv 0, k_{2} \equiv \pm 1$ and $F_{i} u=u(i=1,2)$ equation (18) reduces to second kind autoconvolution equations which are dealt with by Bukhgeim [1].

Example 2. In the theory of viscoelasticity one may propose nonlinear stress-strain relations of the form

$$
\begin{aligned}
\sigma(t)=E \varepsilon(t) & +\int_{0}^{t} k_{1}(t-s) \varepsilon(s) d s \\
& +\int_{0}^{t} k_{0}(s) k_{2}(t-s) F_{1}(\varepsilon(t-s)) F_{2}(\varepsilon(s)) d s
\end{aligned}
$$

where $\sigma$ is the stress, $\varepsilon$ the strain, and $E>0$ a constant. The determination of $\varepsilon$ by given $\sigma$ then is a special case of equation (18) with kernels depending on one variable only. Under corresponding assumptions (19) and (20) we have a bi-unique relationship between $\sigma, \varepsilon$ in $L_{p}(0, T)(p>1)$ and $C[0, T)$ in any finite interval $[0, T]$ (also a relation of form (21) with $\sigma$ and $\varepsilon$ changed may be given as first relation).

Another model equation with this - also from the physical viewpoint - important property is described by the relation

$$
\sigma=E \varepsilon+m_{0} * \varepsilon+m_{1} * m_{2} * m_{3} * \varepsilon * \varepsilon * \varepsilon
$$

with functions $m_{i}$ in $L_{p}(0, T)$ or $C[0, T](i=0,1,2,3)$. The unique solvability of relation (22) for $\varepsilon$ in these spaces follows from Corollary 1 . See $[2,3]$ for general constitutive relations in nonlinear viscoelasticity.

Example 3. In the theory of inverse problems for identifying memory kernels in linear viscoelasticity and heat conduction (infinite) coupled systems of bilinear Volterra integral equations of the form

$$
m(t)+\lambda \int_{0}^{t} A(t-s) m(s) d s=g(t)
$$

occur where

$$
A(t)=\sum_{k=1}^{n} A_{k}(t)
$$

and $A_{k}=A_{k}[m]$ is the solution of the equation

$$
A_{k}(t)-\int_{0}^{t} n_{k}(t-s) A_{k}(s) d s=f_{k}(t)
$$


with kernel

$$
n_{k}(t)=\int_{0}^{t} l_{k}(t-\tau) m(\tau) d \tau=\int_{0}^{t} l_{k}(\tau) m(t-\tau) d \tau \quad(k=1, \ldots, n)
$$

(see, in particular, [8] and also [7, 9]). For $l_{k} \in L_{1}(0, T)$ one has $\left\|n_{k}\right\|_{\sigma, \infty} \leq N_{k}\|m\|_{\sigma, \infty}$ for some constants $N_{k}$, and

$$
\begin{gathered}
\left\|A_{k}[m]\right\|_{\infty, \sigma} \leq e^{T\left\|n_{k}\right\|_{\infty, \sigma}}\left\|f_{k}\right\|_{\infty, \sigma} \\
\left\|A_{k}\left[m_{1}\right]-A_{k}\left[m_{2}\right]\right\|_{\infty, \sigma} \leq T e^{T\left\|n_{k}^{1}\right\|_{\infty, \sigma}}\left\|A_{k}\left[m_{2}\right]\right\|_{\infty, \sigma}\left\|n_{k}^{1}-n_{k}^{2}\right\|_{p, \sigma}
\end{gathered}
$$

where $n_{k}^{i}$ are related to $m_{i}(i=1,2)$. Hence the Theorem with $G_{0}=0, G_{1}(m)=A[m]$ and $G_{2}(m)=m$ yields the unique solvability of equation (23) for $m$ in $L_{\infty}(0, T)$ or $C[0, T]$ for any $g, f_{k}$ in $L_{\infty}(0, T)$ or $C[0, T]$, respectively.

In equation (25) the right-hand side may depend also on $m$, namely $f_{k}=f_{k}(m, t)$, where $f_{k}(0, t)$ in $L_{\infty}(0, T)$ or $C[0, T]$, respectively, and $f_{k}$ fulfills a Lipschitz condition with respect to $m$.

Example 4. The convolution equation of first kind

$$
\int_{0}^{t} y(t-s) x(s) d s=g(t)
$$

where $g \in C^{1}[0, T]$ with $g(0)=0$ and $y$ is solution of the problem

$$
\begin{aligned}
\dot{y}-A(t) y & =f(x)(t) \\
y(0) & =c \neq 0
\end{aligned}
$$

with $A \in L_{\infty}(0, T)$, can be reduced to the second kind equation

$$
c x(t)+\int_{0}^{t}(f(x)(s)+A(s) y(s)) x(t-s) d s=\dot{g}(t)
$$

by differention. In equation (29) there is

$$
y(t)=c \exp \left(-\int_{0}^{t} A(s) d s\right)+\int_{0}^{t} \exp \left(\int_{s}^{t} A(\tau) d \tau\right) f(x)(s) d s .
$$

If the function $f$ : satisfies a Lipschitz condition, then also the operator $y[x]$ in $C[0, T]$ does it and by Corollary 2 we obtain existence and uniqueness of a solution $x \in C[0, T]$. We point out that the more difficult autoconvolution equation of first kind is considered in [4]. 
Example 5. As further example we consider an integro-differential equation of the form

$$
\dot{u}(t)+f(u(t), t)+\int_{0}^{t} k(t, s) F_{1}(u, \dot{u})(t-s) F_{2}(u, \dot{u})(s) d s=g(t)
$$

with $u(0)=c$, where $k \in L_{\infty}([0, T] \times[0, T])$ or $k \in C([0, T] \times[0, T]), f(0, \cdot) \in L_{p}(0, T)$ and $f, F_{i}$ fulfill the Lipschitz conditions

$$
\begin{aligned}
\left|f\left(u_{1}, t\right)-f\left(u_{2}, t\right)\right| & \leq N\left|u_{1}-u_{2}\right| \quad\left(u_{1}, u_{2} \in \mathbb{R}\right) \\
\left|F_{i}\left(u_{1}, v_{1}\right)-F_{i}\left(u_{2}, v_{2}\right)\right| & \leq N_{i}\left(\left|u_{1}-u_{2}\right|+\left|v_{1}-v_{2}\right|\right) \quad\left(u_{1}, u_{2}, v_{1}, v_{2} \in \mathbb{R}\right)
\end{aligned}
$$

with constants $N$ and $N_{i}(i=1,2)$. Writing

$$
u(t)=c+\int_{0}^{t} v(s) d s, \quad v=\dot{u}
$$

in (31), we obtain an equation for $v$. In this equation from (32) and (33) we have

$$
\begin{gathered}
\left\|f\left(u_{1}, \cdot\right)-f\left(u_{2}, \cdot\right)\right\|_{p, \sigma} \leq N\left\|u_{1}-u_{2}\right\|_{p, \sigma} \\
\left\|F_{i}\left(u_{1}, v_{1}\right)-F_{i}\left(u_{2}, v_{2}\right)\right\|_{p, \sigma} \leq N_{i}\left(\left\|u_{1}-u_{2}\right\|_{p, \sigma}+\left\|v_{1}-v_{2}\right\|_{p, \sigma}\right) .
\end{gathered}
$$

From (33) by Young's inequality there follows

$$
\left\|u_{1}-u_{2}\right\|_{p, \sigma} \leq \min \left(T, \frac{1}{\sigma}\right)\left\|v_{1}-v_{2}\right\|_{p, \sigma}
$$

Therefore, the operators $G_{i} v=F_{i}(u, v)(i=1,2)$ satisfy assumption (5) and the operator $G_{0} v=f(u, \cdot)$ fulfills assumption $(6)$ with $\lambda(\sigma)=\frac{1}{\sigma}$. Corollary 2 yields existence and uniqueness of a solution $v$ in $L_{p}(0, T)(p>1)$ or $C[0, T]$, i.e. $u$ in $W_{p}^{1}(0, T)$ or $C^{1}[0, T]$, to equation (31) for any $g$ in $L_{p}(0, T)$ or $C[0, T]$, respectively.

We remark that in Examples 4 and 5 the Lipschitz continuous function $f$ of $x$ or $u$ may depend on $x(h(t))$ or $u(h(t))$, respectively, where it is assumed that $h \in C^{1}[0, T]$ with $\dot{h}(t) \geq \delta>0$ in case $p<\infty, h \in C[0, T]$ in case $p=\infty$, and in both cases $h$ satisfies $0 \leq h(t) \leq t$ in $[0, T]$.

Example 6. In analogous way as (30) integral equations of the form

$$
u(t)+f\left(J_{\alpha_{0}} u(t), t\right)+\int_{0}^{t} k(t, s) F_{1}\left(u, J_{\alpha_{1}} u\right)(t-s) F_{2}\left(u, J_{\alpha_{2}} u\right)(s) d s=g(t)
$$

and integro-differential equations of the form

$$
\begin{aligned}
& \dot{u}(t)+f\left(D_{\beta_{0}} u(t), u(t), J_{\alpha_{0}} u(t), t\right) \\
& \quad+\int_{0}^{t} k(t, s) F_{1}\left(\dot{u}, D_{\beta_{1}} u, u, J_{\alpha_{1}} u\right)(t-s) F_{2}\left(\dot{u}, D_{\beta_{2}} u, u, J_{\alpha_{2}} u\right)(s) d s=g(t) \\
& u(0)=c
\end{aligned}
$$


with operators of fractional integration

$$
J_{\alpha_{k}} u(t)=\frac{1}{\Gamma\left(\alpha_{k}\right)} \int_{0}^{t}(t-s)^{\alpha_{k}-1} u(s) d s \quad\left(0<\alpha_{k}<1 ; k=0,1,2\right)
$$

and operators of fractional differentiation

$$
D_{\beta_{k}} u(t)=\frac{d}{d t} J_{1-\beta_{k}} u(t) \quad\left(0<\beta_{k}<\frac{1}{p} ; k=0,1,2\right)
$$

can be handled (if $c=0$ in (35) only $0<\beta_{k}<1$ is required.) There holds the estimation

$$
\left\|J_{\alpha_{k}} u\right\|_{p, \sigma} \leq \frac{1}{\Gamma\left(\alpha_{k}\right)}\left\|t^{\alpha_{k}-1}\right\|_{1, \sigma}\|u\|_{p, \sigma} \leq \frac{1}{\sigma^{\alpha_{k}}}\|u\|_{p, \sigma}
$$

Further we have

$$
D_{\beta_{k}} u=\frac{1}{\Gamma(1-\beta)} u(0) t^{-\beta_{k}}+J_{1-\beta_{k}} \dot{u} \in L_{p}(0, T) \quad \text { if } \quad \dot{u} \in L_{p}(0, T)
$$

and if $u_{1}(0)=u_{2}(0)=c$, then

$$
\left\|D_{\beta_{k}} u_{1}-D_{\beta_{k}} u_{2}\right\|_{p, \sigma}=\left\|J_{1-\beta_{k}}\left(\dot{u}_{1}-\dot{u}_{2}\right)\right\|_{p, \sigma} \leq \frac{1}{\sigma^{1-\beta_{k}}}\left\|\dot{u}_{1}-\dot{u}_{2}\right\|_{p, \sigma} .
$$

Hence if $k \in L_{\infty}([0, T] \times[0, T])$ or $k \in C([0, T] \times[0, T])$ and $f(0, \cdot) \in L_{p}(0, T)$ or $f(0,0,0, \cdot) \in L_{p}(0, T)$, respectively, and $f, F_{1}, F_{2}$ fulfill uniform Lipschitz conditions with respect to the dependent variables, then equations (34) and (35) have a unique solution $u$ in $L_{p}(0, T) \quad(p>1)$ or $C[0, T]$ and $u$ in $W_{p}^{1}(0, T) \quad(p>1)$ or $C^{1}[0, T]$, respectively, for any $g$ in $L_{p}(0, T)$ or $C[0, T]$.

To an equation of form (35) the following integro-differential equation of first kind can be reduced by differentiation:

$$
\begin{aligned}
& \int_{0}^{t}\left(\dot{u}(s)+a_{1}(t, s) D_{\beta_{1}} u(s)+a_{2}(t, s) u(s)+a_{3}(t, s) J_{\alpha_{1}} u(s)\right) \\
& \quad \times\left(u(t-s)+b(t, s) J_{\alpha_{2}} u(t-s)\right) d s=f(t), \quad u(0)=c \neq 0
\end{aligned}
$$

where $a_{i}$ in $L_{\infty}([0, T] \times[0, T])$ or $C([0, T] \times[0, T]) \quad(i=1,2,3), b$ and $b_{t}$ in $L_{\infty}([0, T] \times$ $[0, T])$ or $C([0, T] \times[0, T]), f$ in $W_{p}^{1}(0, T)(p>1)$ or $C^{1}[0, T]$. 


\section{References}

[1] Bukhgeim, A. L.: Inverse problems of memory reconstruction. J. Inv. Ill-Posed Probl. 1 (1993), 193 - 205.

[2] Christensen, R. M.: Theory of Viscoelasticity. New York: Academic Press 1982.

[3] Findley, W. N, Lai, J. S. and K. Onaran: Creep and Relaxation of Nonlinear Viscoelastic Materials. Amsterdam: North-Holland Publ. Comp. 1976.

[4] Gorenflo, R. and B. Hofmann: On autoconvolution and regularization. Inv. Probl. 10 (1994), 353 - 373.

[5] Gripenberg, G., London, S.-O. and O. Staffans: Volterra Integral and Functional Equations. Cambridge: University Press 1990.

[6] Janno; J.: On an inverse problem for a model of radially wave propagation in the media with memory. In: Numerical Methods and Optimization, Vol. 2 (eds.: G. Vainikko et al). Tallinn: Eston. Acad. Sci. 1990, pp. 4 - 19.

[7] Janno, J.: Global existence for a hyperbolic integro-differential inverse problem. Forum Math. (submitted).

[8] Janno, J. and L. v. Wolfersdorf: Inverse problems for identification of memory kernels in heat flow. J. Inv. Ill-Posed Probl. (submitted).

[9] Wolfersdorf, L. v.: On identification of memory kernels in linear viscoelasticity. Math. Nachr. 161 (1993), 203 - 217.

Received 14.12.1994 